\title{
Finite Volume Evolution Galerkin (FVEG) Methods for Three-Dimensional Wave Equation System
}

\author{
M. Lukáčová-Medvid’ová ${ }^{1}$, G. Warnecke ${ }^{2}$ and Y. Zahaykah²,3
}

\begin{abstract}
The subject of the paper is the derivation of finite volume evolution Galerkin schemes for three-dimensional wave equation system. The aim is to construct methods which take into account all of the infinitely many directions of propagation of bicharacteristics. The idea is to evolve the initial function using the characteristic cone and then to project onto a finite element space. Numerical experiments are presented to demonstrate the accuracy and the multidimensional behaviour of the solutions. Moreover, we construct further new EG schemes by neglecting the so-called source term, i.e. we mimic Kirchhoff's formula. The numerical test shows that such schemes are more accurate and some of them are of second order.
\end{abstract}

Key words: hyperbolic systems, wave equation, evolution Galerkin schemes, recovery stage, finite volume.

\section{Introduction}

Evolution Galerkin methods, EG-methods, were proposed to approximate evolutionary problems of first order hyperbolic systems. These schemes were introduced by Morton for scalar problems and one-dimensional systems, see [2, 3]. In [11] Ostkamp generalized these schemes to approximate the solution of the wave equation system as well as the Euler equations of gas dynamics in two space dimensions. In [4] Lukáčová, Morton and Warnecke new EGschemes with better accuracy and stability were constructed. Further in [12] the approximate evolution operator of the wave equation system in three space dimensions as well as other two-dimensional evolution Galerkin schemes were derived. These methods were applied to the Maxwell and to the Euler equations of gas dynamics, see [9], [6],[8]. Higher order finite volume evolution Galerkin (FVEG) methods have been introduced and studied in [5], [7], [8], [10].

The main objective of this paper is the derivation and analysis of FVEG schemes for the wave equation system in three space dimensions. The bicharacteristics theory of linear hyperbolic systems is used to obtain the exact integral equations. Applying numerical quadratures we derive the approximate evolution operators. Projecting these operators on a finite element space of piecewise constants we obtain the first order evolution Galerkin schemes.

The outline of this paper is as follows: in the next section we briefly describe the general

\footnotetext{
${ }^{1}$ TU Hamburg-Harburg, Arbeitsbereich Mathematik, Schwarzenbergstrasse 95, 21073 Hamburg, Germany, email: lukacova@tu-harburg.de

${ }^{2}$ Institut für Analysis und Numerik, Otto-von-Guericke-Universität Magdeburg, Universitätsplatz 2, 39106 Magdeburg, Germany, emails: Gerald.Warnecke@mathematik.uni-magdeburg.de, Yousef.Zahaykah@mathematik.uni-magdeburg.de

${ }^{3}$ Department of Mathematics, Al Quds University, Jerusalem, Palestine
} 
theory used to derive the exact integral equations. In Section 3 we introduce the finite volume evolution Galerkin schemes. The exact integral equations as well as the approximate evolution operators for the three-dimensional wave equation system are given in Section 4. The derivation of the first order schemes is given in Section 5. In Section 6 numerical tests, which demonstrate accuracy and multidimensionality of our schemes, are presented. Finally in Section 7 we define further new FVEG schemes based on neglecting of the so-called source term, we test and discuss their accuracy.

\section{General Theory}

In this section we recall the derivation of the exact integral equations for a general linear hyperbolic system using the concept of bicharacteristics. The general form of the linear hyperbolic system is given as

$$
\mathbf{u}_{t}+\sum_{k=1}^{d} \mathcal{A}_{k} \mathbf{u}_{x_{k}}=0, \mathbf{x}=\left(x_{1}, \ldots, x_{d}\right)^{T} \in \mathbb{R}^{d}
$$

where the coefficient matrices $\mathcal{A}_{k}, k=1, \ldots, d$ are elements of $\mathbb{R}^{p \times p}$ and the dependent variables are $\mathbf{u}=\left(u_{1}, \ldots, u_{p}\right)^{T}=\mathbf{u}(\mathbf{x}, t) \in \mathbb{R}^{p}$. Let $\mathcal{A}(\mathbf{n})=\sum_{k=1}^{d} n_{k} \mathcal{A}_{k}$ be the pencil matrix, where $\mathbf{n}=\left(n_{1}, \ldots, n_{d}\right)^{T}$ is a directional vector in $\mathbb{R}^{d}$. The matrix $\mathcal{A}(\mathbf{n})$ has $p$ real eigenvalues $\lambda_{k}, k=$ $1, \ldots, p$ and $p$ corresponding linearly independent right eigenvectors $\mathbf{r}_{k}=\mathbf{r}_{k}(\mathbf{n}), k=1, \ldots, p$. Let $\mathcal{R}=\left[\mathbf{r}_{1}\left|\mathbf{r}_{2}\right| \ldots \mid \mathbf{r}_{p}\right]$ be the matrix of right eigenvectors then we can define the characteristic variable $\mathbf{w}=\mathbf{w}(\mathbf{n})$ as $\partial \mathbf{w}(\mathbf{n})=\mathcal{R}^{-1} \partial \mathbf{u}$. Since system (2.1) has constant coefficient matrices $\mathcal{A}_{k}$ we have $\mathbf{w}=\mathcal{R}^{-1} \mathbf{u}$ or $\mathbf{u}=\mathcal{R} \mathbf{w}$. Multiplying equation (2.1) by $\mathcal{R}^{-1}$ from the left we get

$$
\mathcal{R}^{-1} \mathbf{u}_{t}+\sum_{k=1}^{d} \mathcal{R}^{-1} \mathcal{A}_{k} \mathcal{R} \mathcal{R}^{-1} \mathbf{u}_{x_{k}}=0 .
$$

Let $\mathcal{B}_{k}=\mathcal{R}^{-1} \mathcal{A}_{k} \mathcal{R}=\left(b_{i j}^{k}\right)_{i, j=1}^{p}$, where $k=1,2, \ldots, d$, then the equation (2.2) can be rewritten in the following form

$$
\mathbf{w}_{t}+\sum_{k=1}^{d} \mathcal{B}_{k} \mathbf{w}_{x_{k}}=0 .
$$

Let us introduce the decomposition $\mathcal{B}_{k}=\mathcal{D}_{k}+\mathcal{B}_{k}^{\prime}$, where $\mathcal{D}_{k}$ contains the diagonal part of the matrix $\mathcal{B}_{k}$. This yields

$$
\mathbf{w}_{t}+\sum_{k=1}^{d} \mathcal{D}_{k} \mathbf{w}_{x_{k}}=-\sum_{k=1}^{d} \mathcal{B}_{k}^{\prime} \mathbf{w}_{x_{k}}=: \mathbf{s} .
$$

The $i$-th bicharacteristic corresponding to the $i$-th equation of (2.3) is defined by

$$
\frac{d \mathbf{x}_{i}}{d \tilde{t}}=\mathbf{b}_{i i}(\mathbf{n})=\left(b_{i i}^{1}, b_{i i}^{2}, \ldots, b_{i i}^{d}\right)^{T},
$$

where $i=1, \ldots, p$. Here $b_{i i}^{k}$ are the diagonal entries of the matrix $\mathcal{B}_{k}, k=1, \ldots, d, i=1, \ldots, p$. 


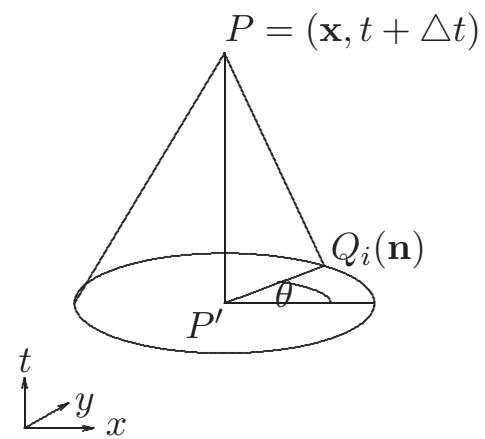

Figure 1: Bicharacteristics along the Mach cone through $P$ and $Q_{i}(\mathbf{n}), d=2$.

We consider the bicharacteristics backwards in time. Therefore the initial conditions are $\mathbf{x}_{i}(t+\Delta t, \mathbf{n})=\mathbf{x}$ for all $\mathbf{n} \in \mathbb{R}^{d}$ and $i=1, \ldots, p$, i.e. $\mathbf{x}_{i}(\tilde{t}, \mathbf{n})=\mathbf{x}-\mathbf{b}_{i i}(\mathbf{n})(t+\Delta t-\tilde{t})$.

We will integrate the $i$-th equation of the system (2.3) from the point $\mathrm{P}$ down to the point $Q_{i}(\mathbf{n}):=Q_{i}\left(\mathbf{x}_{i}(\mathbf{n}, t), t\right)$, where the bicharacteristic hits the basic plane, see Figure 1 . Note that bicharacteristics are straight lines because the system is linear with constant coefficients. Now the $i$-th equation reads

$$
\frac{\partial w_{i}}{\partial t}+\sum_{k=1}^{d} b_{i i}^{k} \frac{\partial w_{i}}{\partial x_{k}}=-\left(\sum_{j=1, i \neq j}^{d}\left(b_{i j}^{1} \frac{\partial w_{j}}{\partial x_{1}}+b_{i j}^{2} \frac{\partial w_{j}}{\partial x_{2}}+\ldots+b_{i j}^{d} \frac{\partial w_{j}}{\partial x_{d}}\right)\right)=s_{i},
$$

where $P \equiv(\mathbf{x}, t+\Delta t) \in \mathbb{R}^{p} \times \mathbb{R}_{+}$is taken to be a fixed point, while $Q_{i}(\mathbf{n})=\left(\mathbf{x}-\Delta t \mathbf{b}_{i i}, t\right)$. Taking a vector $\sigma_{i}=\left(b_{i i}^{1}, b_{i i}^{2}, \ldots, b_{i i}^{d}, 1\right)$, we can define the directional derivative

$$
\frac{d w_{i}}{d \sigma_{i}}=\left(\frac{\partial w_{i}}{\partial x_{1}}, \frac{\partial w_{i}}{\partial x_{2}}, \ldots, \frac{\partial w_{i}}{\partial x_{d}}, \frac{\partial w_{i}}{\partial t}\right) \cdot \sigma_{i}=\frac{\partial w_{i}}{\partial t}+b_{i i}^{1} \frac{\partial w_{i}}{\partial x_{1}}+b_{i i}^{2} \frac{\partial w_{i}}{\partial x_{2}}+\ldots+b_{i i}^{d} \frac{\partial w_{i}}{\partial x_{d}} .
$$

Hence the $i$-th equation (2.4) can be rewritten as follows

$$
\frac{d w_{i}}{d \sigma_{i}}=s_{i}=-\sum_{j=1, i \neq j}^{d}\left(b_{i j}^{1} \frac{\partial w_{j}}{\partial x_{1}}+b_{i j}^{2} \frac{\partial w_{j}}{\partial x_{2}}+\ldots+b_{i j}^{d} \frac{\partial w_{j}}{\partial x_{d}}\right) .
$$

Now the integration from $P$ to $Q_{i}(\mathbf{n})$ gives

$$
w_{i}(P)-w_{i}\left(Q_{i}(\mathbf{n}), \mathbf{n}\right)=s_{i}^{\prime},
$$

where

$$
s_{i}^{\prime}=\int_{t}^{t+\Delta t} s_{i}\left(\mathbf{x}_{i}(\tilde{t}, \mathbf{n}), \tilde{t}\right) d \tilde{t}=\int_{0}^{\Delta t} s_{i}\left(\mathbf{x}_{i}(\tau, \mathbf{n}), t+\Delta t-\tau\right) \mathrm{d} \tau .
$$

Multiplication of equation $(2.5)$ by $\mathcal{R}$ from the left and $(d-1)$-dimensional integration of the variable $\mathbf{n}$ over the unit sphere $O$ in $\mathbb{R}^{d}$ leads to the exact integral equations for (2.1)

$$
\mathbf{u}(P)=\mathbf{u}(\mathbf{x}, t+\Delta t)=\frac{1}{|O|} \int_{O} \mathcal{R}(\mathbf{n})\left(\begin{array}{c}
w_{1}\left(Q_{1}(\mathbf{n}), \mathbf{n}\right) \\
w_{2}\left(Q_{2}(\mathbf{n}), \mathbf{n}\right) \\
w_{3}\left(Q_{3}(\mathbf{n}), \mathbf{n}\right) \\
\vdots \\
w_{p}\left(Q_{p}(\mathbf{n}), \mathbf{n}\right)
\end{array}\right) \mathrm{d} O+\tilde{\mathbf{s}}
$$


where

$$
\tilde{\mathbf{s}}=\left(\tilde{s}_{1}, \tilde{s}_{2}, \ldots, \tilde{s}_{p}\right)^{T}=\frac{1}{|O|} \int_{O} \mathcal{R}(\mathbf{n}) \mathbf{s}^{\prime} \mathrm{d} O=\frac{1}{|O|} \int_{O} \int_{0}^{\Delta t} \mathcal{R}(\mathbf{n}) \mathbf{s}\left(\mathbf{x}_{i}(\tau, \mathbf{n}), t+\Delta t-\tau\right) \mathrm{d} \tau \mathrm{d} O
$$

and $|O|$ corresponds to the measure of the domain of integration.

\section{Evolution Galerkin Schemes}

In this section we recall the definition of the finite volume evolution Galerkin (FVEG) schemes. We assume that $d=3$ and $\Delta x, \Delta y, \Delta z>0$ are the mesh size parameters in the $x-, y-, z-$ direction, respectively. We construct a regular mesh, which consists of the mesh cells

$$
\begin{aligned}
\Omega_{k l m} & =\left[\left(k-\frac{1}{2}\right) \Delta x,\left(k+\frac{1}{2}\right) \Delta x\right] \times\left[\left(l-\frac{1}{2}\right) \Delta y,\left(l+\frac{1}{2}\right) \Delta y\right] \times\left[\left(m-\frac{1}{2}\right) \Delta z,\left(m+\frac{1}{2}\right) \Delta z\right] \\
& =\left[x_{k}-\frac{\Delta x}{2}, x_{k}+\frac{\Delta x}{2}\right] \times\left[y_{l}-\frac{\Delta y}{2}, y_{l}+\frac{\Delta y}{2}\right] \times\left[z_{m}-\frac{\Delta z}{2}, z_{m}+\frac{\Delta z}{2}\right],
\end{aligned}
$$

where $k, l, m \in \mathbb{Z}$. Suppose that $S_{h}^{q}$ is a finite element space consisting of piecewise polynomials of order $q \geq 0$ with respect to the mesh and assume constant time step, i.e. $t_{n}=n \Delta t$. Let $\mathbf{U}^{n}$ be an approximation in the space $S_{h}^{q}$ to the exact solution $\mathbf{u}\left(., t_{n}\right)$ at time $t_{n} \geq 0$. We denote by $R_{h}: S_{h}^{q} \rightarrow S_{h}^{r}$ a recovery operator, $r>q \geq 0$ and by $E_{\tau}$ the so-called approximate evolution operator, which is a suitable approximation to the exact integral equations (2.6). In the next section such approximate evolution operators are derived for the wave equation system.

Definition 3.1 Starting from some initial data $\mathbf{U}^{0} \in S_{h}^{q}$, the finite volume evolution Galerkin method (FVEG) is recursively defined by means of

$$
\mathbf{U}^{n+1}=\mathbf{U}^{n}-\int_{0}^{\Delta t} \sum_{j=1}^{3} \frac{1}{\Delta_{x_{j}}} \delta_{x_{j}} \mathbf{f}_{j}\left(\tilde{\mathbf{U}}^{n+\frac{\tau}{\Delta t}}\right) d \tau,
$$

where we use equivalent notation for the space variables $(x, y, z)=\left(x_{1}, x_{2}, x_{3}\right)$ and denote by $\delta_{x_{j}} \mathbf{f}_{j}\left(\tilde{\mathbf{U}}^{n+\frac{\tau}{\Delta t}}\right)$ an approximation to the face flux difference and $\delta_{x}$ is defined by $\delta_{x}=v(x+$ $\left.\frac{\Delta x}{2}\right)-v\left(x-\frac{\Delta x}{2}\right)$. The cell boundary value $\tilde{\mathbf{U}}^{n+\frac{\tau}{\Delta t}}$ is evolved using the approximate evolution operator $E_{\tau}$ to $t_{n}+\tau$ and averaged along the cell boundary, i.e.

$$
\tilde{\mathbf{U}}^{n+\frac{\tau}{\Delta t}}=\sum_{k, l, m \in \mathbb{Z}}\left(\frac{1}{\left|\partial \Omega_{k l m}\right|} \int_{\partial \Omega_{k l m}} E_{\tau} R_{h} \mathbf{U}^{n} d S\right) \chi_{\partial \Omega_{k l m}}
$$

where $\chi_{\partial \Omega_{k l m}}$ is the characteristic function of $\partial \Omega_{k l m}$.

It is important to note that in the updating step (3.2) some numerical quadratures are used instead of the exact time integration. In this paper we are using the midpoint rule for the time integration in (3.2). Similarly, to evaluate the intermediate value $\tilde{\mathbf{U}}^{n+\frac{\tau}{\Delta t}}$ in (3.3) the three dimensional integrals along the cell face are evaluated by means of suitable numerical quadratures, e.g. the trapezoidal rule is used in the numerical experiments below. Integrals aroud the unit sphere $O$, which arrive from $E_{\tau}$ are evaluated exactly. In this way all of the infinitely many directions of wave propagations are taken into account explicitly. 


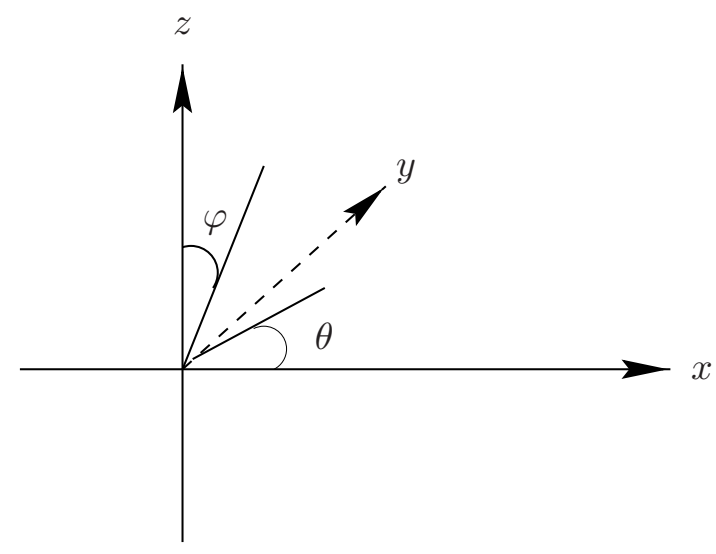

Figure 2: Spherical coordinates.

\section{Exact Integral Equations and Approximate Evolution Op- erators for the Wave Equation System}

In this section we derive two first order approximate evolution operators for the threedimensional wave equation system. We begin by applying the general theory given in Section 2. It is well known that the wave equation can be written as a first order system of partial differential equations in the form

$$
\begin{aligned}
& \phi_{t}+c \nabla \cdot \mathbf{v}=0, \\
& \mathbf{v}_{t}+c \nabla \phi=0,
\end{aligned}
$$

where $c$ is a constant, speed of sound, and $\mathbf{v}(\mathbf{x}, t):=(u, v, w)^{T}(\mathbf{x}, t),(\mathbf{x}, t) \in \mathbb{R}^{3} \times \mathbb{R}^{+}$is the velocity field. Let $\mathbf{u}:=(\phi, \mathbf{v})^{T}$ be the vector of the unknown variables and let the fluxes be $\mathbf{f}_{1}(\mathbf{u}):=(c u, c \phi, 0,0)^{T}, \mathbf{f}_{2}(\mathbf{u}):=(c v, 0, c \phi, 0)^{T}$ and $\mathbf{f}_{3}(\mathbf{u}):=(c w, 0,0, c \phi)^{T}$. The system (4.1) can be written in the conservation form

$$
\frac{\partial \mathbf{u}}{\partial t}+\sum_{k=1}^{3} \frac{\partial \mathbf{f}_{k}(\mathbf{u})}{\partial x_{k}}=0
$$

Using Jacobian matrices of the flux functions we can rewrite system (4.2) in the linearized form

$$
\mathbf{u}_{t}+\mathcal{A}_{1} \mathbf{u}_{x}+\mathcal{A}_{2} \mathbf{u}_{y}+\mathcal{A}_{3} \mathbf{u}_{z}=0 .
$$

Let $\mathbf{n}:=\left(n_{1}, n_{2}, n_{3}\right)^{T}:=(\cos \theta \sin \varphi, \sin \theta \sin \varphi, \cos \varphi)^{T}$ be a directional vector in $\mathbb{R}^{3}$ with $\theta \in[0,2 \pi]$ and $\varphi \in[0, \pi]$, see Fig 2. Then the system (4.3) has four real eigenvalues $\lambda_{1}=$ $-c, \lambda_{2}=0, \lambda_{3}=0, \lambda_{4}=c$ and four corresponding right eigenvectors

$$
\mathbf{r}_{1}=\left(\begin{array}{c}
-1 \\
\mathbf{n}
\end{array}\right), \mathbf{r}_{2}=\left(\begin{array}{c}
0 \\
n_{2} \\
-n_{1} \\
0
\end{array}\right), \mathbf{r}_{3}=\left(\begin{array}{c}
0 \\
n_{3} \\
0 \\
-n_{1}
\end{array}\right), \mathbf{r}_{4}=\left(\begin{array}{l}
1 \\
\mathbf{n}
\end{array}\right) .
$$


The vector of characteristic variables $\mathbf{w}=\left(w_{1}, w_{2}, w_{3}, w_{4}\right)^{T}$ is given as

$$
\mathbf{w}(\mathbf{n})=\mathcal{R}^{-1}(\mathbf{n}) \mathbf{u}=\left[\begin{array}{c}
\frac{1}{2} \mathbf{u} \cdot \mathbf{r}_{1} \\
\frac{1}{n_{1}^{2}} \mathbf{u} \cdot\left[\left(n_{1}^{2}+n_{3}^{2}\right) \mathbf{r}_{2}-n_{2} n_{3} \mathbf{r}_{3}\right] \\
\frac{1}{n_{1}^{2}} \mathbf{u} \cdot\left[-n_{2} n_{3} \mathbf{r}_{2}+\left(n_{1}^{2}+n_{2}^{2}\right) \mathbf{r}_{3}\right] \\
\frac{1}{2} \mathbf{u} \cdot \mathbf{r}_{4}
\end{array}\right]
$$

To determine the points $Q_{i}(\mathbf{n}), i=1,2,3,4$, recall that the bicharacteristics $\mathbf{x}_{i}$, which correspond to this system are given as $\frac{d \mathbf{x}_{i}}{d \tilde{t}}=\mathbf{b}_{i i}=\left(b_{i i}^{1}, b_{i i}^{2}, b_{i i}^{3}\right)^{T}, i=1,2,3,4$. Hence we get $\mathbf{x}_{i}(\mathbf{n}, \tilde{t})=\mathbf{x}-\mathbf{b}_{i i}(t+\Delta t-\tilde{t})$. Therefore we have for the footpoints of the bicharacteristics $Q_{i}\left(\mathbf{x}_{i}(\mathbf{n}, t), t\right)=\left(\mathbf{x}-\mathbf{b}_{i i} \Delta t, t\right)$. From the diagonal matrices $\mathcal{D}_{1}, \mathcal{D}_{2}, \mathcal{D}_{3}$, see Section 2 , we get $\mathbf{b}_{11}=-c \mathbf{n}, \mathbf{b}_{22}=\mathbf{b}_{33}=\mathbf{0}$ and $\mathbf{b}_{44}=c \mathbf{n}$. Hence

$$
\begin{array}{rll}
Q_{2} \equiv Q_{3} & \text { correspond to } & \lambda_{2,3}=0 \\
Q_{1} & \text { corresponds to } & \lambda_{1}=-c \\
Q_{4} & \text { corresponds to } & \lambda_{4}=c
\end{array}
$$

In the following two lemmas we give some invariance properties for terms in spherical coordinates. Let $f$ be a continuous function. Consider the unit sphere

$$
O=\left\{(x, y, z):\left(x-x_{0}\right)^{2}+\left(y-y_{0}\right)^{2}+\left(z-z_{0}\right)^{2}=1\right\} .
$$

Then the integral of $f$ over $O$ satisfies the following property:

\section{Lemma 4.5}

$$
\int_{0}^{2 \pi} \int_{\pi}^{2 \pi} f(x, y, z) \sin \varphi d \varphi d \theta=-\int_{0}^{2 \pi} \int_{0}^{\pi} f(x, y, z) \sin \varphi d \varphi d \theta .
$$

Proof: The proof follows directly from the fact that the mappings

$$
\begin{aligned}
& {[0,2 \pi] \times[\pi, 2 \pi] \rightarrow O \subset \mathbb{R}^{3},(\theta, \varphi) \mapsto\left(x_{0}+\cos \theta \sin \varphi, y_{0}+\sin \theta \sin \varphi, z_{0}+\cos \varphi\right),} \\
& {[0,2 \pi] \times[\pi, 2 \pi] \rightarrow O \subset \mathbb{R}^{3},(\theta, \varphi) \mapsto\left(x_{0}-\cos \theta \sin \varphi, y_{0}-\sin \theta \sin \varphi, z_{0}-\cos \varphi\right)}
\end{aligned}
$$

are two parameterizations of the same unit sphere $O$.

Corollary 4.6 Let $a \in \mathbb{R}$ be a constant. Then

1. $\int_{0}^{2 \pi} \int_{\pi}^{2 \pi}(a+x) f(x, y, z) \sin \varphi d \varphi d \theta=-\int_{0}^{2 \pi} \int_{0}^{\pi}(a+x) f(x, y, z) \sin \varphi d \varphi d \theta$.

2. $\int_{0}^{2 \pi} \int_{\pi}^{2 \pi}(a+y) f(x, y, z) \sin \varphi d \varphi d \theta=-\int_{0}^{2 \pi} \int_{0}^{\pi}(a+y) f(x, y, z) \sin \varphi d \varphi d \theta$.

3. $\int_{0}^{2 \pi} \int_{\pi}^{2 \pi}(a+z) f(x, y, z) \sin \varphi d \varphi d \theta=-\int_{0}^{2 \pi} \int_{0}^{\pi}(a+z) f(x, y, z) \sin \varphi d \varphi d \theta$.

Proof: These properties follow from Lemma 4.5 by taking $g=\left(a+x^{\prime}\right) f(x, y, z)$, where $x^{\prime} \in\{x, y, z\}$. 
Lemma 4.7 Consider the characteristic variables $w_{1}$ and $w_{4}$ and the points $Q_{1}$ and $Q_{4}$. Then

1. $\sin (\pi+\varphi) w_{4}\left(Q_{4}(\theta, \pi+\varphi), \theta, \pi+\varphi\right)=\sin \varphi w_{1}\left(Q_{1}(\theta, \varphi), \theta, \varphi\right)$.

2. $\sin (\pi+\varphi) \sin (\pi+\varphi) w_{4}\left(Q_{4}(\theta, \pi+\varphi), \theta, \pi+\varphi\right)=-\sin \varphi \sin \varphi w_{1}\left(Q_{1}(\theta, \varphi), \theta, \varphi\right)$.

3. $\cos (\pi+\varphi) \sin (\pi+\varphi) w_{4}\left(Q_{4}(\theta, \pi+\varphi), \theta, \pi+\varphi\right)=-\cos \varphi \sin \varphi w_{1}\left(Q_{1}(\theta, \varphi), \theta, \varphi\right)$.

Proof: Using the symmetry between the points $Q_{1}$ and $Q_{4}$ we obtain

$$
\begin{aligned}
w_{4}\left(Q_{4}(\theta, \pi+\varphi), \theta, \pi+\varphi\right) & =\frac{1}{2}\left[\phi\left(Q_{4}(\theta, \pi+\varphi)\right)+\cos \theta \sin (\pi+\varphi) u\left(Q_{4}(\theta, \pi+\varphi)\right)\right. \\
& \left.+\sin \theta \sin (\pi+\varphi) v\left(Q_{4}(\theta, \pi+\varphi)\right)+\cos (\pi+\varphi) w\left(Q_{4}(\theta, \pi+\varphi)\right)\right] \\
= & \frac{1}{2}\left[\phi\left(Q_{1}(\theta, \varphi)\right)-\cos \theta \sin \varphi u\left(Q_{1}(\theta, \varphi)\right)-\sin \theta \sin \varphi v\left(Q_{1}(\theta, \varphi)\right)\right. \\
& \left.-\cos \varphi w\left(Q_{1}(\theta, \varphi)\right)\right]=-w_{1}\left(Q_{1}(\theta, \varphi), \theta, \varphi\right) .
\end{aligned}
$$

Then the three properties follow directly from equation (4.8).

In the next lemma we give the integral equations that are equivalent to the wave equation system in three dimensions (4.1). Let $\mathcal{J}=\left[\mathbf{J}_{0}\left|\mathbf{J}_{1}\right| \mathbf{J}_{2} \mid \mathbf{J}_{3}\right]$ be the matrix whose columns are the vectors $\mathbf{J}_{0}=(0,0,0)^{T}, \mathbf{J}_{1}=(-1,0,0)^{T}+n_{1} \mathbf{n}, \mathbf{J}_{2}=(0,-1,0)^{T}+n_{2} \mathbf{n}, \mathbf{J}_{3}=(0,0,-1)^{T}+n_{3} \mathbf{n}$. Set $\tilde{P}=(\mathbf{x}, t+\Delta t-\tau), \tilde{Q}=(\mathbf{x}+c \tau \mathbf{n}, t+\Delta t-\tau), P=(\mathbf{x}, t+\Delta t), Q=(\mathbf{x}+c \Delta t \mathbf{n}, t)$ and $P^{\prime}=(\mathbf{x}, t)$, where $\tau \in[0, \Delta t]$.

Lemma 4.9 The exact integral representation (3.2) for the wave equation system (4.1) reads

$$
\mathbf{u}(P)=\left(\frac{d-1}{d}\right) \mathbf{u}^{\prime}\left(P^{\prime}\right)+\frac{1}{|O|} \int_{O} \mathbf{u}(Q) \cdot(-1, \mathbf{n})^{T}(-1, \mathbf{n})^{T} d S+\tilde{\mathbf{s}},
$$

where $d$ is the dimension, i.e. $d=3$ in our case, $\mathbf{u}^{\prime}\left(P^{\prime}\right)=(0, \mathbf{v})^{T}\left(P^{\prime}\right)$ and

$$
\begin{aligned}
& \tilde{\mathbf{s}}=\frac{1}{|O|} \int_{O} \int_{0}^{\Delta t}\left((-1, \mathbf{n})^{T} s(\tilde{Q})+c \nabla \phi(\tilde{P}) \mathcal{J}\right) \mathrm{d} \tau \mathrm{d} S \\
& s=\frac{c}{1-n_{3}^{2}}\left(n_{2}^{2} u_{x}-n_{1} n_{2}\left(u_{y}+v_{x}\right)+n_{1}^{2} v_{y}\right)+\frac{1}{\tau} \frac{d \mathbf{n}}{d \varphi} \cdot \frac{d \mathbf{v}}{d \varphi}, \\
& c \nabla \phi(\tilde{P}) \mathcal{J}=\left(c \nabla \phi \cdot \mathbf{J}_{0}, c \nabla \phi \cdot \mathbf{J}_{1}, c \nabla \phi \cdot \mathbf{J}_{2}, c \nabla \phi \cdot \mathbf{J}_{3}\right)^{T} .
\end{aligned}
$$

Proof: See[12].

Remark 4.13 Since the point $\tilde{P}$ is independent of the directional vector $\mathbf{n}$ we can evaluate the second part of the integral (4.11) to get $\left(\frac{1-d}{d}\right)\left(\mathbf{u}^{\prime}\left(P^{\prime}\right)-\mathbf{u}^{\prime}(P)\right)$. Then substituting in the 
equation (4.10) and rearranging the terms we get the equivalent system of the exact integral equations

$$
\mathbf{u}(P)=\frac{k}{|O|} \int_{O} \mathbf{u}(Q) \cdot(-1, \mathbf{n})^{T}(-1, \mathbf{n})^{T} d S+k \tilde{\mathbf{s}},
$$

where $\tilde{\mathbf{s}}$ is given as

$$
\tilde{\mathbf{s}}=\frac{1}{|O|} \int_{O} \int_{0}^{\Delta t}(-1, \mathbf{n})^{T} s(\tilde{Q}) d \tau d S
$$

$s$ is given in the equation (4.12). For the first component of the vector $\mathbf{u}$, i.e. for $\phi$, we have $k=1$, otherwise $k=d$.

To obtain explicit numerical methods we approximate time integrals in equations (4.11) and (4.15) using the backward rectangle rule, which gives

$$
\begin{aligned}
& \tilde{\mathbf{s}}=\frac{\Delta t}{|O|} \int_{O}\left((-1, \mathbf{n})^{T} s(Q)+c \nabla \phi\left(P^{\prime}\right) \mathcal{J}\right) \mathrm{d} \tau \mathrm{d} S+\mathcal{O}\left(\Delta t^{2}\right) \\
& \tilde{\mathbf{s}}=\frac{\Delta t}{|O|} \int_{O}(-1, \mathbf{n})^{T} s(Q) \mathrm{d} \tau \mathrm{d} S+\mathcal{O}\left(\Delta t^{2}\right)
\end{aligned}
$$

respectively. In order to evaluate the integral of the source terms $s$ we need the following lemma. The proof can be found in [12].

\section{Lemma 4.18}

$$
\begin{aligned}
\Delta t \int_{O} s(Q) d S & =\int_{O}\left(2 n_{1} u(Q)+2 n_{2} v(Q)+2 n_{3} w(Q)\right) d S \\
\Delta t \int_{O} n_{1} s(Q) d S & =\int_{O}\left(\left(-1+3 n_{1}^{2}\right) u(Q)+3 n_{1} n_{2} v(Q)+3 n_{1} n_{3} w(Q)\right) d S \\
\Delta t \int_{O} n_{2} s(Q) d S & =\int_{O}\left(3 n_{1} n_{2} u(Q)+\left(-1+3 n_{2}^{2}\right) v(Q)+3 n_{2} n_{3} w(Q)\right) d S, \\
\Delta t \int_{O} n_{3} s(Q) d S & =\int_{O}\left(3 n_{1} n_{3} u(Q)+3 n_{2} n_{3} v(Q)+\left(-1+3 n_{3}^{2}\right) w(Q)\right) d S
\end{aligned}
$$

Using the Taylor theorem we can approximate the integral of the second term in the equation (4.16), which yields

$$
\begin{aligned}
\int_{O} c \nabla \phi\left(P^{\prime}\right) \cdot \mathbf{J}_{1} \mathrm{~d} S & =\int_{O}-2 n_{1} \phi(Q) \mathrm{d} S+\mathcal{O}\left(\Delta t^{2}\right), \\
\int_{O} c \nabla \phi\left(P^{\prime}\right) \cdot \mathbf{J}_{2} \mathrm{~d} S & =\int_{O}-2 n_{2} \phi(Q) \mathrm{d} S+\mathcal{O}\left(\Delta t^{2}\right), \\
\int_{O} c \nabla \phi\left(P^{\prime}\right) \cdot \mathbf{J}_{3} \mathrm{~d} S & =\int_{O}-2 n_{3} \phi(Q) \mathrm{d} S+\mathcal{O}\left(\Delta t^{2}\right) .
\end{aligned}
$$


Let $\mathcal{I}(m)=\left[\mathbf{I}_{0}\left|\mathbf{I}_{1}\right| \mathbf{I}_{2} \mid \mathbf{I}_{3}\right], m \in\{1,3\}$, be the matrix with the column vectors

$$
\begin{aligned}
& \mathbf{I}_{0}=(0,0,0,0)^{T}+(1,-3 \mathbf{n})^{T} \\
& \mathbf{I}_{1}=(0,-1,0,0)^{T}+n_{1}(-m, 4 \mathbf{n})^{T} \\
& \mathbf{I}_{2}=(0,0,-1,0)^{T}+n_{2}(-m, 4 \mathbf{n})^{T} \\
& \mathbf{I}_{3}=(0,0,0,-1)^{T}+n_{3}(-m, 4 \mathbf{n})^{T}
\end{aligned}
$$

Substituting equations (4.19-4.25) in the equation (4.10) we get the following approximate evolution operator, which we call the EG3 for 3D, see [6]

$$
\mathbf{u}(P)=\left(\frac{d-1}{d}\right) \mathbf{u}^{\prime}\left(P^{\prime}\right)+\frac{1}{|O|} \int_{O} \mathbf{u}(Q) \mathcal{I}(3) \mathrm{d} S+\mathcal{O}\left(\Delta t^{2}\right)
$$

where $\mathbf{U}(Q) \mathcal{I}(3)=\phi(Q) \mathbf{I}_{0}+u(Q) \mathbf{I}_{1}+v(Q) \mathbf{I}_{2}+w(Q) \mathbf{I}_{3}$.

Remark 4.27 Substituting equations (4.19-4.22) in the equation (4.14) we get the following approximate evolution operator

$$
\mathbf{u}(P)=\frac{k}{|O|} \int_{O} \mathbf{u}(Q) \mathcal{I}(1) d S+\mathcal{O}\left(\Delta t^{2}\right),
$$

where $\mathbf{U}(Q) \mathcal{I}(1)$ is defined analogously as $\mathbf{U}(Q) \mathcal{I}(3)$. This approximate evolution operator is analog to the two-dimensional approximate operator EG1, see [6].

\section{$5 \quad$ Numerical Schemes and Discretisation}

This section is devoted to the numerical experiments using the finite volume EG scheme based on the approximate evolution operator (4.26) and on 3.1. Analogous to the two-dimensional case, cf. [12], we use the midpoint rule to approximate the time integral in the equation (3.2) and the trapezoidal rule to approximate the cell face integrals given in (3.3). Let $\tilde{\mathbf{U}}_{l}$ denote the intermediate value corresponding to the left side of the cell $i j k$, see Figure 3. Further, let $\tilde{\mathbf{U}}_{f}$ and $\tilde{\mathbf{U}}_{b}$ be the intermediate values corresponding to the front and to the bottom sides, respectively. Let $N_{x}, N_{y}$ and $N_{z}$ be the number of cells along the $x-, y-$ and $z$-axis, respectively; recall that $\Delta x, \Delta y, \Delta z$ are the mesh steps in the corresponding directions. Then the first order evolution Galerkin algorithm is formulated as follows:

- input of the initial data $\mathbf{U}^{0}$.

- determine the time step $\Delta t$ from the CFL condition $\frac{c \Delta t}{\min \{\Delta x, \Delta y, \Delta z\}}=\nu<1$.

- do the time loop

- calculate the intermediate values $\tilde{\phi}_{l}, \tilde{u}_{l}, \tilde{\phi}_{f}, \tilde{v}_{f}, \tilde{\phi}_{b}, \tilde{w}_{b}$ according (3.3) using the approximate evolution operator EG3-3D, cf. (4.26) 
- update:

$$
\begin{aligned}
\phi_{i j k}^{n+1}= & \phi_{i}^{n}-\frac{c \Delta t}{\Delta x}\left(\tilde{u}_{l}(i+1 j k)-\tilde{u}_{l}(i j k)\right)-\frac{c \Delta t}{\Delta y}\left(\tilde{v}_{f}(i j+1 k)-\tilde{v}_{f}(i j k)\right) \\
& \quad-\frac{c \Delta t}{\Delta z}\left(\tilde{w}_{b}(i j k+1)-\tilde{w}_{b}(i j k)\right) \\
u_{i j k}^{n+1}= & u_{i}^{n}-\frac{c \Delta t}{\Delta x}\left(\tilde{\phi}_{l}(i+1 j k)-\tilde{\phi}_{l}(i j k)\right) \\
v_{i j k}^{n+1}= & v_{i}^{n}-\frac{c \Delta t}{\Delta y}\left(\tilde{\phi}_{f}(i j+1 k)-\tilde{\phi}_{f}(i j k)\right) \\
w_{i j k}^{n+1}= & w_{i}^{n}-\frac{c \Delta t}{\Delta z}\left(\tilde{\phi}_{b}(i j k+1)-\tilde{\phi}_{b}(i j k)\right) .
\end{aligned}
$$

- carry out the boundary conditions.

- end of the time loop.

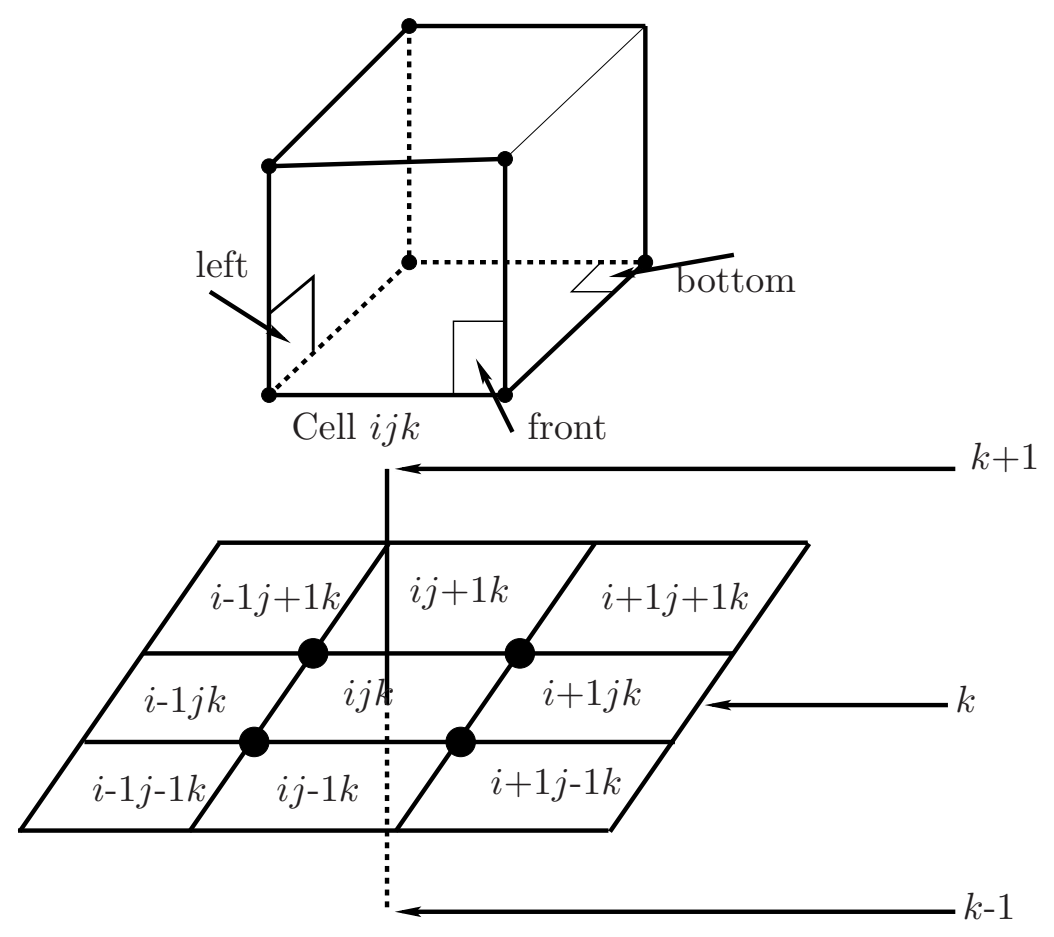

Figure 3: Cell ijk and its neighbours

This is the so-called FVEG3-3D scheme, note that analogous numerical schemes based on the approximate evolution operator (4.28) can be derived. Finally, we note that in the experiments presented below we evaluate the intermediate values $\tilde{\mathbf{U}}$ on cell face by using the piecewise constant approximate functions, i.e. the scheme is first order in space. 


\section{$6 \quad$ Numerical Tests}

\section{Example 6.1}

Consider the three dimensional wave equation system together with the periodic boundary conditions and the initial data

$$
\begin{aligned}
& \phi(x, y, 0)=-(\sin (2 \pi x)+\sin (2 \pi y)+\sin (2 \pi z)) \\
& u(x, y, 0)=v(x, y, 0)=w(x, y, 0)=0 .
\end{aligned}
$$

where $(x, y, z) \in[-1,1] \times[-1,1] \times[-1,1]$. The exact solution is

$$
\begin{aligned}
\phi(x, y, t) & =-\cos (2 \pi t)(\sin (2 \pi x)+\sin (2 \pi y)+\sin (2 \pi z)) \\
u(x, y, t) & =\sin (2 \pi t) \cos (2 \pi x), \\
v(x, y, t) & =\sin (2 \pi t) \cos (2 \pi y), \\
w(x, y, t) & =\sin (2 \pi t) \cos (2 \pi z) .
\end{aligned}
$$

The following two tables show the $L^{2}$-error and the experimental order of convergence (EOC), which is defined using two solutions computed on meshes of sizes $N_{1}, N_{2}$, as follows

$$
\mathrm{EOC}=\ln \frac{\left\|\mathbf{U}_{N_{1}}(T)-\mathbf{U}_{N_{1}}^{n}\right\|}{\left\|\mathbf{U}_{N_{2}}(T)-\mathbf{U}_{N_{2}}^{n}\right\|} / \ln \left(\frac{N_{2}}{N_{1}}\right) .
$$

The numerical experiments are carried out with the FVEG3-3D scheme. We take the final time $T=0.1$ and $T=0.2$, respectively, and set the constant $c$ equal to 1 . Moreover we consider a uniform mesh, i.e. we take the mesh size $h>0, h=\Delta x=\Delta y=\Delta z$, and set the CFL number to be 0.5. The last column of Tables 1 and 2 demonstrates that the experimental order of convergence is 1 .

\begin{tabular}{|c|c|c|c|c|}
\hline $\mathrm{N}$ & $\left\|\phi(T)-\phi^{n}\right\|$ & $\left\|u(T)-u^{n}\right\|$ & $\left\|\mathbf{U}(T)-\mathbf{U}^{n}\right\|$ & $\mathrm{EOC}$ \\
\hline 20 & 0.55892402464 & 0.16838548872 & 0.63044197493 & \\
\hline 40 & 0.27817890698 & 0.09911167224 & 0.32688358021 & 0.9476 \\
\hline 80 & 0.13874739504 & 0.05362621166 & 0.16696751589 & 0.9692 \\
\hline 160 & 0.06927101055 & 0.02788768195 & 0.08444904569 & 0.9834 \\
\hline 320 & 0.03460647659 & 0.01422055263 & 0.04247682396 & 0.9914 \\
\hline
\end{tabular}

Table 1: FVEG3-3D scheme, $\mathrm{T}=0.1, \mathrm{CFL}=0.5$

In Figures 4 we plot the first and the second components of the solution $\phi(x, 0.5,0.5, T)$ and $u(x, 0.5,0.5, T)$ (on top and on bottom, respectively) restricted to a horizontal line for a $80 \times 80 \times 80$ and a $320 \times 320 \times 320$ mesh at the end time $T=0.2$. The exact solution is plotted as well. We can notice a good agreement between the approximate and the exact solutions.

\section{Example 6.4}

It is easy to see that the wave equation system preserves exactly the vorticity, i.e. the vector $\left(\frac{\partial u}{\partial y}-\frac{\partial v}{\partial x}, \frac{\partial u}{\partial z}-\frac{\partial w}{\partial x}, \frac{\partial v}{\partial z}-\frac{\partial w}{\partial y}\right)^{T}$ is constant in time. Components of vorticity vector are 

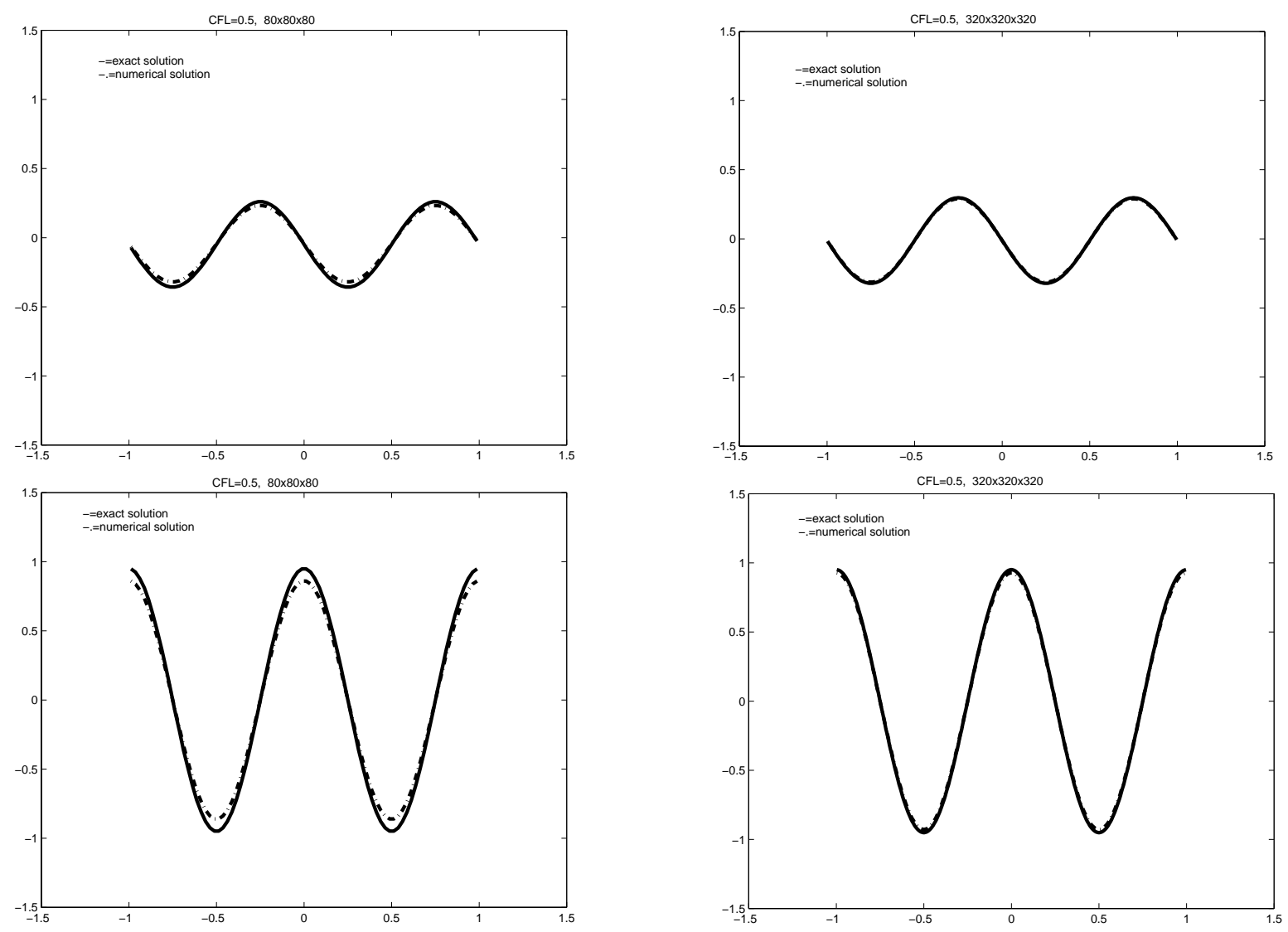

Figure 4: Solution of the wave equation system computed using the $80 \times 80 \times 80$ mesh cells (left) and $320 \times 320 \times 320$ mesh cells (right); top: $\phi(x, 0.5,0.5,0.2)$, bottom: $u(x, 0.5,0.5,0.2)$. 


\begin{tabular}{|c|c|c|c|c|}
\hline $\mathrm{N}$ & $\left\|\phi(T)-\phi^{n}\right\|$ & $\left\|u(T)-u^{n}\right\|$ & $\left\|\mathbf{U}(T)-\mathbf{U}^{n}\right\|$ & $\mathrm{EOC}$ \\
\hline 20 & 0.49587434262 & 0.59746694762 & 1.14751541347 & \\
\hline 40 & 0.23446528786 & 0.33325251909 & 0.62301339926 & 0.8812 \\
\hline 80 & 0.11224035516 & 0.17664332961 & 0.32589337983 & 0.9349 \\
\hline 160 & 0.05458773142 & 0.09102322692 & 0.16683975527 & 09659 \\
\hline 320 & 0.02686871668 & 0.04621317753 & 0.08443282103 & 0.9826 \\
\hline
\end{tabular}

Table 2: FVEG3-3D scheme, $\mathrm{T}=0.2, \mathrm{CFL}=0.5$

trivially zero in Example 6.1, because $u$ depends on $x$ only, $v$ depends on $y$ only while $w$ depends on $z$ only. In this example we take the following nontrivial initial data for which the exact solution still has vanishing vorticity

$$
\begin{aligned}
& \phi(x, y, 0)=-\exp \left(-10\left(x^{2}+y^{2}+z^{2}\right)\right) \\
& u(x, y, 0)=v(x, y, 0)=w(x, y, 0)=0 .
\end{aligned}
$$

We compute the vector of discrete vorticity given by the formulae:

$$
\begin{aligned}
& \mathrm{DV}_{k l m}^{(1)}=\mu_{x} \delta_{y} u_{k+\frac{1}{2}, l+\frac{1}{2}, m-\frac{1}{2}}-\mu_{y} \delta_{x} v_{k+\frac{1}{2}, l+\frac{1}{2}, m-\frac{1}{2}} \\
& \mathrm{DV}_{k l m}^{(2)}=\mu_{x} \delta_{z} u_{k+\frac{1}{2}, l-\frac{1}{2}, m+\frac{1}{2}}-\mu_{z} \delta_{x} w_{k+\frac{1}{2}, l-\frac{1}{2}, m+\frac{1}{2}} \\
& \mathrm{DV}_{k l m}^{(3)}=\mu_{y} \delta_{z} v_{k-\frac{1}{2}, l+\frac{1}{2}, m+\frac{1}{2}}-\mu_{z} \delta_{y} w_{k-\frac{1}{2}, l+\frac{1}{2}, m+\frac{1}{2}}
\end{aligned}
$$

for each $k, l, m \in \mathbb{Z}$, where we denote by $u_{k+\frac{1}{2}, l+\frac{1}{2}, m-\frac{1}{2}}:=u\left(\left(k-\frac{1}{2}\right) h,\left(l+\frac{1}{2}\right) h,\left(m+\frac{1}{2}\right) h\right)$ the values at the corner point $\left(\left(k-\frac{1}{2}\right) h,\left(l+\frac{1}{2}\right) h,\left(m+\frac{1}{2}\right) h\right)$ of the cubic mesh cell $\Omega_{k m l}$. The other corner values are defined analogously. The $\mu, \delta$-operators are defined, e.g. in the $x-$ direction, as

$$
\delta_{x} f(x)=f\left(x+\frac{h}{2}\right)-f\left(x-\frac{h}{2}\right), \quad \mu_{x} f(x)=\frac{f\left(x+\frac{h}{2}\right)+f\left(x-\frac{h}{2}\right)}{2} ;
$$

operators in other directions are analogous. In Table 3 we show the average value of DV ${ }^{(1)}$ (average values of $\mathrm{DV}^{(2)}$ and $\mathrm{DV}^{(3)}$ are same due to the symmetry of the problem) at time $T=0.2$ using $N=50, N=100$ and $N=200$ mesh cells in each direction. The computational domain is a cube $[-1,1]^{3}$ and $\mathrm{CFL}=0.55$. The results in this table indicate that the FVEG3$3 \mathrm{D}$ preserves the discrete vorticity given by the equation (6.6). Further in the Figures 5 and 6 we plot the surface of the solution $\phi$ for the cross section $z=0.5$ and the isosurface of $\phi$, respectively.

\begin{tabular}{|c|c|c|c|}
\hline Mesh size & $50 \times 50 \times 50$ & $100 \times 100 \times 100$ & $200 \times 200 \times 200$ \\
\hline Average vorticity & $1.871 \times 10^{-5}$ & $6.480 \times 10^{-6}$ & $3.350 \times 10^{-6}$ \\
\hline
\end{tabular}

Table 3: Vorticity preservation for the EG3-3D scheme 


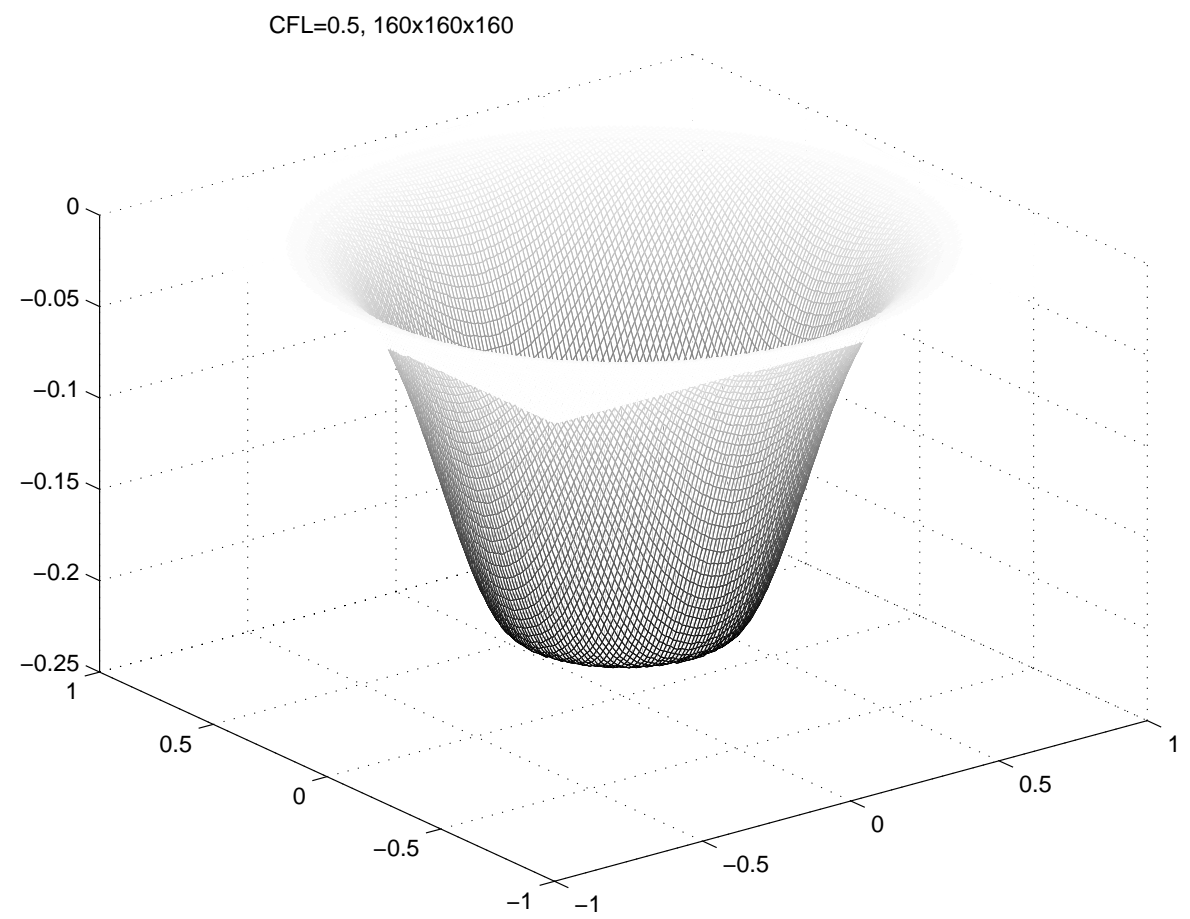

Figure 5: Cross section surfaces $(z=0.5)$ of the approximated solution $\phi$ at $T=0.2$ using $16 \times 160$ mesh cells.

$\mathrm{CFL}=0.5,160 \times 160 \times 160$

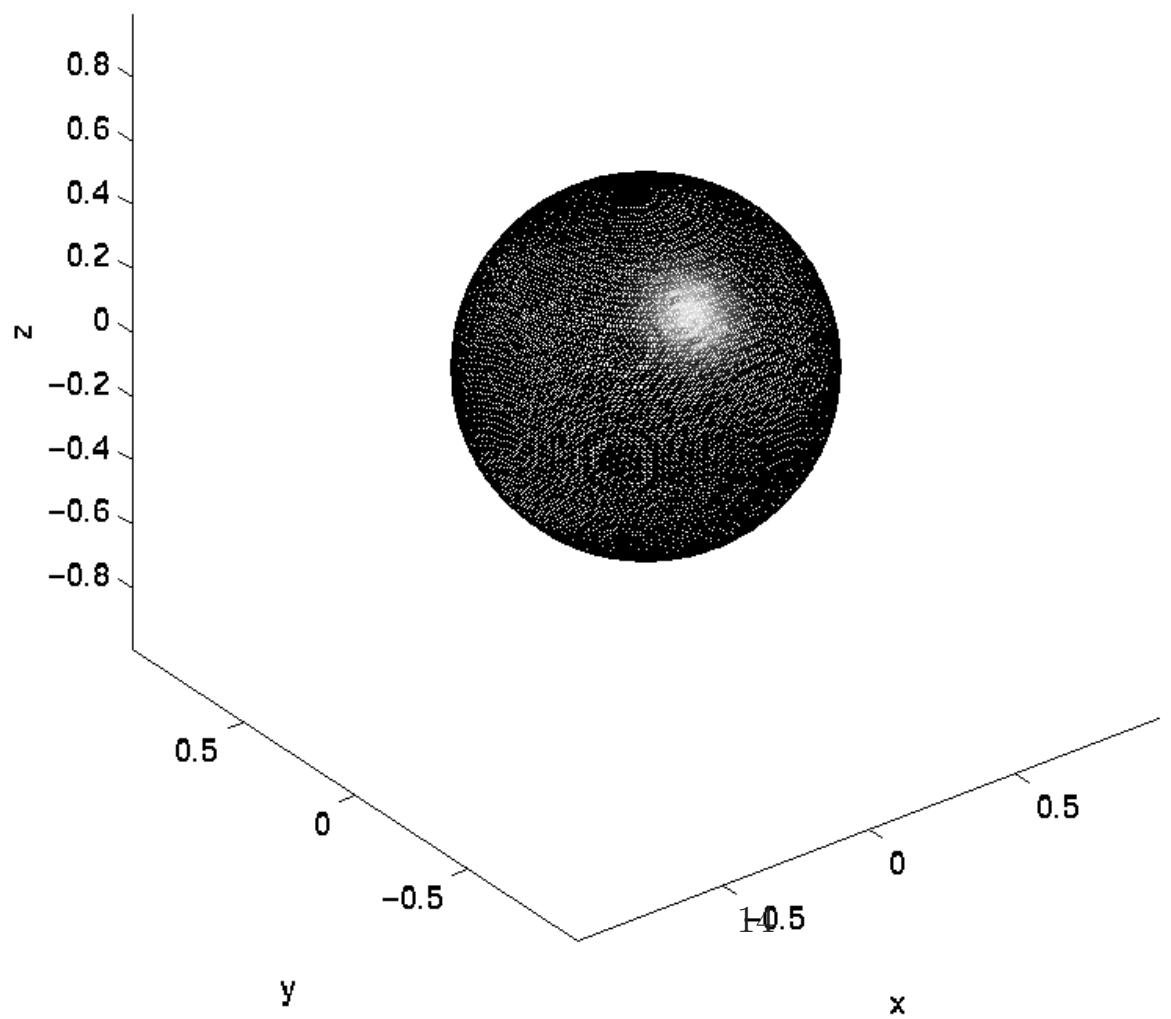

Figure 6: Isosurface of the approximated solution $\phi(x, y, z, 0.2)$ using $16 \times 160$ mesh cells. 


\section{Further EG schemes}

It is well known that the solution of the wave equation in a space of odd dimension $(>2)$ depends on the initial data distributed on the surface of the sphere centered at the observation point (Kirchhoff's formula), see [1]. We follow this concept and ignore the $\tilde{\mathbf{s}}$ part appearing in the exact integral equations (4.10) and (4.14). This leads us to the following approximate evolution operators:

$$
\begin{aligned}
& \mathbf{u}(P)=\left(\frac{d-1}{d}\right) \mathbf{u}^{\prime}\left(P^{\prime}\right)+\frac{1}{|O|} \int_{O} \mathbf{u}(Q) \cdot(-1, \mathbf{n})^{T}(-1, \mathbf{n})^{T} \mathrm{~d} S, \\
& \mathbf{u}(P)=\frac{k}{|O|} \int_{O} \mathbf{u}(Q) \cdot(-1, \mathbf{n})^{T}(-1, \mathbf{n})^{T} \mathrm{~d} S .
\end{aligned}
$$

We call these N1 and N2 approximate evolution operators, respectively. Now, we apply the numerical schemes based on N1 and N2 operators to the problem given in the Example 6.1. The $L^{2}$ errors and EOC are given in Tables 4-6 and indicate a superconvergence of the finite volume scheme N1. Actually using piecewise constants we have obtained the overall second order accuracy. Further, Table 6 indicates that the finite volume scheme N2 is of the first order.

We conjecture that the superconvergence phenomenon of the N1 method is due to the fact that the source term $\tilde{\mathbf{s}}$, cf. (4.11), that we have neglected, is actually at least of the order $O\left(\Delta t^{3}\right)$. Moreover, it is important to keep the term $\left(\frac{d-1}{d}\right) \mathbf{u}^{\prime}\left(P^{\prime}\right)$ in (7.1), which increases apparently the order of accuracy. The proof of this fact is still open.

\begin{tabular}{|c|c|c|c|c|}
\hline $\mathrm{N}$ & $\left\|\phi(T)-\phi^{n}\right\|$ & $\left\|u(T)-u^{n}\right\|$ & $\left\|\mathbf{U}(T)-\mathbf{U}^{n}\right\|$ & $\mathrm{EOC}$ \\
\hline 20 & 0.03948481218 & 0.05612849451 & 0.10492985314 & \\
\hline 40 & 0.01297997347 & 0.01347014919 & 0.02669858553 & 1.9746 \\
\hline 80 & 0.00360947268 & 0.00326143223 & 0.00670366419 & 1.9937 \\
\hline 160 & 0.00094540786 & 0.00080017118 & 0.00167768226 & 1.9985 \\
\hline 320 & 0.00024154741 & 0.00019803770 & 0.00041952585 & 1.9996 \\
\hline
\end{tabular}

Table 4: N1 scheme, $\mathrm{T}=0.1, \mathrm{CFL}=0.5$

\begin{tabular}{|c|c|c|c|c|}
\hline $\mathrm{N}$ & $\left\|\phi(T)-\phi^{n}\right\|$ & $\left\|u(T)-u^{n}\right\|$ & $\left\|\mathbf{U}(T)-\mathbf{U}^{n}\right\|$ & $\mathrm{EOC}$ \\
\hline 20 & 0.17589540963 & 0.06529837724 & 0.20911917497 & \\
\hline 40 & 0.04832075618 & 0.01308638944 & 0.05337280435 & 1.9701 \\
\hline 80 & 0.01247555949 & 0.00283409813 & 0.01340656261 & 1.9932 \\
\hline 160 & 0.00315867513 & 0.00065347754 & 0.00335534309 & 1.9984 \\
\hline 320 & 0.00079405157 & 0.00015651727 & 0.00083905355 & 1.9996 \\
\hline
\end{tabular}

Table 5: N1 scheme, $\mathrm{T}=0.2, \mathrm{CFL}=0.5$ 


\begin{tabular}{|c|c|c|c|c|}
\hline $\mathrm{N}$ & $\left\|\phi(T)-\phi^{n}\right\|$ & $\left\|u(T)-u^{n}\right\|$ & $\left\|\mathbf{U}(T)-\mathbf{U}^{n}\right\|$ & $\mathrm{EOC}$ \\
\hline 20 & 0.55892402464 & 0.11225699162 & 0.59177779767 & \\
\hline 40 & 0.28602634464 & 0.05665197288 & 0.30238949704 & 0.9686 \\
\hline 80 & 0.14568931489 & 0.02860078232 & 0.15388109280 & 0.9746 \\
\hline 160 & 0.07365113809 & 0.01439264027 & 0.07775560702 & 0.9848 \\
\hline 320 & 0.03704518272 & 0.00722271923 & 0.03910049337 & 0.9918 \\
\hline
\end{tabular}

Table 6: N2 scheme, $\mathrm{T}=0.1, \mathrm{CFL}=0.5$

\section{Acknowledgements.}

This research was supported by the VolkswagenStiftung Agency, by the Deutsche Forschungsgemeinschaft Grant No. Wa 633/6-2 as well as and by the Graduate College 413 of the University Hamburg and TU Hamburg-Harburg. Authors gratefully acknowledge these supports.

\section{References}

[1] Lawrence C. Evans. Partial Differential Equations. American Mathematical Society, 1998.

[2] P. Lin, K.W. Morton, , and E. Süli. Characteristic Galerkin schemes for scalar conservation laws in two and three space dimensions. SIAM J. Numer. Anal., 34(2):779-796, 1997.

[3] P. Lin, K.W. Morton, and E. Süli. Euler characteristic Galerkin scheme with recovery. $M^{2} A N, 27(7): 863-894,1993$.

[4] M. Lukáčová, K. W. Morton, and G. Warnecke. Finite volume evolution Galerkin methods for Euler equations of gas dynamics. Int. J. Num. Methods in Fluids, 40(3-4):425-434, 2002.

[5] M. Lukáčová, K.W. Morton, and G. Warnecke. High resolution finite volume evolution Galerkin schemes for multidimensional conservation laws. Proceedings of ENNUMAMATH'99, World Scientific Publishing Company, Singapore, 1999.

[6] M. Lukáčová, K.W. Morton, and G. Warnecke. Evolution Galerkin methods for hyperbolic systems in two space dimensions. Math. Comput., 69(232):1355-1384, 2000.

[7] M. Lukáčová, K.W. Morton, and G. Warnecke. Evolution Galerkin methods for multidimensional hyperbolic systems. Proceedings of ECCOMAS 2000, Barcelona, 1-14, 2000 .

[8] M. Lukáčová, J. Saibartová, and G. Warnecke. Finite volume evolution Galerkin methods for nonlinear hyperbolic systems. J. Comput. Phys., 183:533-562, 2002.

[9] M. Lukáčová, J. Saibertová, G. Warnecke, and Y. Zahaykah. On evolution Galerkin methods for the Maxwell and the linearized Euler equations. accepted to Appl. Math., 2003. 
[10] M. Lukáčová, G. Warnecke, and Y. Zahaykah. Third order finite volume evolution Galerkin (FVEG) methods for two-dimensional wave equation system. J. Numer. Math., 11(3):235-251, 2003.

[11] S. Ostkamp. Multidimensional characteristic Galerkin schemes and evolution operators for hyperbolic systems. Math. Meth. Appl. Sci., 20:1111-1125, 1997.

[12] Y. Zahaykah. Evolution Galerkin Schemes and Discrete Boundary Conditions for Multidimensional First Order Systems. Dissertation, Magdeburg, 2002. 